\title{
Effects of different ceramic primers and surface treatments on the shear bond strength of restorative composite resin to zirconium
}

\author{
Masoumeh Hasani Tabatabaei ${ }^{1}$, Nasim Chiniforush ${ }^{2}$, Seyedeh Fatemeh Namdar ${ }^{3}$ \\ 1: Associate Professor of Restorative Dentistry, School of Dentistry, Tehran University of Medical Sciences, \\ Tebran, Iran \\ 2: PhD candidate of laser dentistry, Laser Research Center of Dentistry, Dentistry Research Institute, \\ Tehran University of Medical Sciences, Tehran, Iran \\ 3: Assistant Professor of Operative dentistry, Dental Materials Research Center, \\ Mashbad University of Medical Sciences, Mashbad, Iran
}

\begin{abstract}
Background and aims: Data are limited about the effect of ceramic primers on the bond of zirconia to restorative resin composite. The aim of this study was to assess the effect of different surface treatments and two ceramic primers on shear bond strength (SBS) of zirconia to restorative resin composite.

Materials and methods: 100 samples of zirconium ceramic blocks were randomly divided into 5 groups $(\mathrm{n}=20)$ and received the following surface treatments:

a) Control group

b) $\mathrm{AL}_{2} \mathrm{O}_{3}$ abrasion

c) Nd:YAG laser

d) Er:YAG laser

e) Bur preparation.

Next, each group was divided into two subgroups of 20. In ten out of 20 specimens in each group, monobond plus and Tetric ceram composite resin were applied on the surface. ZPrime Plus and Elite composite were applied on the surfaces of the remaining 10 specimens in each group. Samples were then subjected to shear bond strength test in a universal testing machine until fracture. The mean SBS were calculated and statistically analyzed by two-way ANOVA and T-Test. P $<0.05$ was considered statistically significant.

Results: In ZPrime plus subgroups, diamond bur yielded the highest bond strength. Laser groups showed no significant difference with the control group. In monobond plus subgroups, SBS of air abrasion and diamond bur subgroups was highest and Nd:YAG laser showed the lowest SBS. The lowest bond strength belonged to the Nd:YAG laser subgroup of monobond plus group.

Conclusions: Type of surface preparation significantly affected the bond strength.
\end{abstract}

Key words: Zirconia $\cdot$ Laser Treatment $\cdot$ Air Abrasion $\cdot$ Bond Strength

\section{Introduction}

In recent decades, esthetic demands have led to use of ceramics for indirect dental restorations. Zirconia ceramics are among the newly introduced all-ceramic systems. Previous studies have showed that zirconium dioxide ceramics exhibit optimal mechanical and optical properties as a framework material ${ }^{1}$. On the other hand, one of the

\section{Addressee for Correspondence:}

Seyedeh Fatemeh Namdar.

Address: Dental Materials Research Center,

Mashhad University of Medical Sciences, Mashhad, Iran

Email: F.Namdar90@gmail.com

Phone: +989155081447 Fax: +985138829500 drawbacks of the zirconia fixed partial dentures (FPDs) is the weak bond between zirconia core and veneering ceramic, which increases the risk of chipping of ceramic veneers. Incidence of chipping was $15.2 \%$ after 5 years of function that this failure rate is higher for patients with bruxism and other parafunctional habits ${ }^{2)}$.

Fracture of all-ceramic restorations causes many problems for both clinician and patient because their replacement is associated with patient discomfort, high-cost and possible damage to the remaining tooth structure and is time consuming as well ${ }^{1,3)}$.

Received date: August 13th, 2017 Accepted date: April 20th, 2018 
Alternately, these restorations can be directly repaired using composite resin. Successful performance of this treatment depends on the mechanical integrity and high bond strength of layering composite resin to zirconia framework ${ }^{4}$. Several methods have been suggested for ceramic surface preparation. Micromechanical retentions and rough surfaces can improve the bond strength of zirconia ceramics to resin cements ${ }^{5}$. Sandblasting with alumina particles is among the newly introduced techniques, in which, alumina particles deposit on the substrate surface and cause surface roughness and micromechanical retention ${ }^{6}$. In the other hand, air-borne particle abrasion not only increases mechanical retention but also cleans and activates framework materials ${ }^{7}$. However, difficult handling of alumina particles is one limitation of this method ${ }^{8)}$.Komine et al. proposed that $0.1 \mathrm{MPa}$ or higher provided durable bond strength between zirconia ceramic and indirect resin composite ${ }^{9}$.

Laser irradiation is also used for zirconia surface preparation. Several studies have investigated the effects of laser on zirconia ceramic surface and have suggested laser application as a potential method of causing zirconia surface roughness to enhance the zirconia bond to resin cement ${ }^{10-12)}$. However, Ersu et al. proposed that there is no relationship between surface roughness and shear bond strength of resin composite to zirconia ceramics. They demonstrated that either air abrasion or $\mathrm{CO}_{2}$ laser irradiation can increase mechanical bond strength ${ }^{13)}$.

To enhance the chemical bond, adhesive monomers are used instead of silane. A layer of metal oxide covers the zirconia ceramic surface, allowing adhesive monomers to react with the ceramic surface and chemically increase the bond strength ${ }^{14)}$. Kitayama et al. reported that the use of priming agents containing phosphonic acid or phosphate monomer can improve bond strength between resin composite and zirconia ceramics ${ }^{15)}$.

Data are limited regarding the effect of adhesive monomers on zirconia bond to restorative composite resin. Thus, this study aimed to assess the effect of application of two different primer systems on the bond strength of zirconia to resin composite following zirconia surface preparation with Er:YAG and Nd:YAG lasers, diamond bur and air abrasion (in comparison with a control group). We hypothesized that different surface preparations and adhesive protocols may affect the shear bond strength of composite to zirconia. We also hypothesized that Er:YAG and Nd:YAG lasers can probably increase the bond strength of zirconia to composite resin.

\section{Materials and methods}

A total of 100 pre-sintered zirconia blocks (ICE Zirkonzahn GmbH, Bruneck, Italy) with dimensions of $10 \times 10$ $\times 2 \mathrm{~mm}$ were fabricated by a diamond blade mounted in mecatome T201 low speed cutting saw machine (PRESI,
Grenoble, France). All of the samples were sintered according to the manufacturer's instructions, and immersed in acrylic resin using metal molds. Each specimen was polished using 800, 1000 and 1200 grit silicon carbide with water-cooling. The samples were rinsed with distilled water for 6 minutes by ultrasonic device. Then the samples were randomly divided in 5 groups $(n=20)$ according to surface preparations as follows:

- Group 1: The control group, which the samples were polished without any treatment.

- Group 2: Specimens were airborne-particle abraded using $\mathrm{AL}_{2} \mathrm{O}_{3}(50 \mu), 2.8$ bar pressure for 10 seconds at a distance of $10 \mathrm{~mm}$ from the surface.

- Group 3: The zirconia surface was irradiated using Nd:YAG laser (FidelisIII, Fotona, Slovenia). Laser parameters were set at an energy density of $111.96 \mathrm{~J} /$ $\mathrm{cm}^{2}$, output power of $1.5 \mathrm{~W}$, for 1 minute. Nd:YAG laser was used with a frequency of $10 \mathrm{~Hz}$ and the pulse duration of $100 \mu \mathrm{sec}$ (medium short pulse) at a wavelength of $1064 \mathrm{~nm}$. Total surfaces of the blocks were scanned with $320 \mu$ fiber about $1 \mathrm{~mm}$ above the surface for 1 minute. All laser treatments were done in scanning mode and perpendicularly to the surface.

- Group 4: Er:YAG laser irradiation (Smart 2940D, Deka laser, Florence, Italy) with an energy density of $5.85 \mathrm{~J} / \mathrm{cm}^{2}$ and output power of $2 \mathrm{~W}$ for 10 seconds. Er:YAG laser was used with a wavelength of 2940 $\mathrm{nm}$, pulse duration of $230 \mu \mathrm{sec}$ (very short pulse) and frequency of $10 \mathrm{~Hz}$. The spot size was $1 \mathrm{~mm}$ and the handpiece was used at a distance of $4 \mathrm{~mm}$ (focusing distance of the handpiece) above the surface (non-contact mode) for 10 seconds accompanied with water and air spray.

- Group 5: Bur preparation with 010 medium grit diamond fissure bur, high speed handpiece with air and water spray, 5 sweep motion.

\section{Shear bond strength assessment:}

Next, each group was divided into 2 subgroups of 10 . In 10 out of 20 specimens in each group, a layer of monobond plus (Ivoclar Vivadent AG.Liechtenstein) was applied on $9 \mathrm{~mm}^{2}$ of the surface according to the manufacturer's instructions and an air spray was used to evaporate the solvent. A plastic tube (Tygon tube) with an internal diameter and height of $3 \mathrm{~mm}$ was placed on the bonding area of the ceramic surface and Tetric ceram composite resin (Ivoclar Vivadent AG.Liechtenstein) was incrementally applied to the tube. Each layer was cured for 40 seconds using a light curing unit (Guilin woodpecker medical instrument Co. China) with a light intensity of $650 \mathrm{~mW} / \mathrm{cm}^{2}$. Z Prime Plus (BISCO INC, Schaumberg, USA) was applied on the surfaces of the remaining 10 specimens in each group according to the manufacturer's instructions. Elite composite (BISCO INC, Schaum- 
berg, USA) was applied in $2 \mathrm{~mm}$ increments and light cured. Chemical composition of the materials used in this study is shown in Table 1. Plastic tubes were then cut by a scalpel. Samples were then subjected to shear bond strength tests in a universal testing machine (Zwick ROELL Z2.5 MA 18-1-3/7 Ulm, Germany) at a crosshead speed of $0.5 \mathrm{~mm} / \mathrm{min}$ under $2.5 \mathrm{kN}$ load until fracture. The mean shear bond strength and standard deviation values were calculated and statistically analyzed.

Data were analyzed using SPSS version 20 (Microsoft, IL, USA), one-way ANOVA and two-way ANOVA. T-test was used for pairwise comparison of groups. $\mathrm{P} \leq$ 0.05 was considered statistically significant.

\section{Results}

One-way ANOVA showed that in Z Prime plus subgroups, surface preparation had a significant effect on the shear bond strength, and surface roughening by diamond bur yielded the highest bond strength among all subgroups. Air abrasion and control subgroups ranked second and the lowest value belonged to Er:YAG laser group. Although the bond strength value following bur preparation was significantly different from the values in the two laser groups $(\mathrm{P}<0.05)$, the mean bond strength after diamond bur preparation was not significantly different from the value in air abrasion group $(\mathrm{P}>0.05)$. On the other hand, Er:YAG and Nd:YAG laser groups showed no significant difference with the control group in terms of bond strength $(\mathrm{P}>0.05)$. (table. 2)

One-way ANOVA showed that in monobond plus subgroups, the shear bond strength of air abrasion and diamond bur subgroups was not significantly different ( $\mathrm{P}$ $>0.05$ ). However, the mentioned two subgroups had a significantly higher bond strength than the control, Er:YAG and Nd:YAG laser subgroups( $\mathrm{P}<0.05)$. Of these, Nd:YAG laser subgroup showed the lowest bond strength.

Two-way ANOVA showed that among all, the lowest bond strength belonged to the Nd:YAG laser subgroup of monobond plus group while the highest value was seen in the diamond bur preparation subgroup of $\mathrm{Z}$ Prime Plus group. (table2)

Table 1: Name and chemical composition of used materials in this study.

\begin{tabular}{|c|c|}
\hline Brand & Chemical composition \\
\hline ZPrime plus & $\begin{array}{l}\text { - BPDM (Biphenyl dimethacrylate) } \\
\text { - 10-MDP (10-methacryloxydecyl dihydrogen phosphate) } \\
\text { - Ethanol }\end{array}$ \\
\hline Monobond plus & $\begin{array}{l}\text { - Silane methacrylate } \\
\text { - 10-MDP } \\
\text { - Sulphide methacrylate } \\
\text { - Ethanol }\end{array}$ \\
\hline Resin composite $Æ$ lite ${ }^{\mathrm{TM}}$ & $\begin{array}{l}\text { - Bis-EMA (bisphenol-A ethoxy dimethacrylate) } \\
\text { - Bis-GMA (Bisphenol A diglycidylmethacrylate) } \\
\text { - TEGDMA (Triethyleneglycol dimethacrylate) } \\
\text { - Glass frit, amorphous silica } 74 \text { vol\% }\end{array}$ \\
\hline Resin composite Tetric ceram & $\begin{array}{l}\text { - Bis-GMA } \\
\text { - TEGDMA } \\
\text { - UDMA } \\
\text { - Silanated barium glass filler } \\
\text { - Ba-Al-F-Si-glass ytterbium tri fluoride } \\
\text { - Highly dispersed silicon dioxide } \\
\text { - Spheroid mixed oxide: } 78.6 \% \mathrm{wt}\end{array}$ \\
\hline
\end{tabular}

Table 2: the mean $\pm S D$ of shear bond strength $(\mathrm{MPa})$ of the study groups (The same letters indicate no significant differences between groups)

\begin{tabular}{|c|c|c|}
\hline group primer & ZPrimer Plus & Monobond plus \\
\hline Control & $13.34 \pm 4.83^{\mathrm{a}, \mathrm{b}}$ & $11.99 \pm 2.43^{\mathrm{a}}$ \\
\hline Air Abrasion & $16.36 \pm 3.11^{\mathrm{a}, \mathrm{b}}$ & $17.52 \pm 2.31^{b}$ \\
\hline Nd:YAG laser & $12.36 \pm 4.35^{\mathrm{a}}$ & $8.34 \pm 1.45^{b}$ \\
\hline Er:YAG laser & $11.59 \pm 2.86^{\mathrm{a}}$ & $11.41 \pm 2.01^{\mathrm{a}}$ \\
\hline Diamond Bur grinding & $18.73 \pm 4.93^{b}$ & $17.83 \pm 3.01^{\mathrm{b}}$ \\
\hline
\end{tabular}


Pairwise comparison of groups with t-test revealed that Er:YAG laser preparation, air abrasion and diamond bur preparation caused no significant difference between the two groups of monobond plus and Z Prime Plus( $\mathrm{P}>$ 0.05). However, after Nd:YAG laser preparation, the difference between the two primers was statistically signifi$\operatorname{cant}(\mathrm{P}<0.05)$. In other words, $Z$ Prime Plus Nd:YAG laser subgroup showed significantly higher bond strength than monobond plus Nd:YAG laser subgroup.

\section{Discussion}

This study assessed the effect of different surface preparations and type of primers on shear bond strength of zirconia ceramic to composite resin. Bond strength to zirconia ceramic is influenced by some factors such as micromechanical retention, chemical bonds, concentration of defects at the interface and wetting properties of the veneering layer ${ }^{11}$. Mechanical roughening processes for feldspathic, leucite and lithium disilicate ceramics are based on etching by HF acid ${ }^{17)}$. Moreover, application of silane can increase the chemical bond between resin and ceramic because silane, via the siloxane bonds, is capable of chemically bonding to the silica content of ceramic ${ }^{18)}$. However, oxide ceramics such as zirconia and alumina require more advanced techniques for higher bond strength since they have low silica content ${ }^{19-21)}$. Furthermore, since the irregularities caused during the process of manufacturing and milling of ceramic do not provide adequately high bond strength, surface preparations seem to be necessary for bonding of the composite resin to these ceramics ${ }^{22}$. Mechanical surface roughening by diamond bur and air abrasion using aluminum oxide particles are among the commonly used methods to increase micromechanical retention. By one swiping motion of the diamond bur over the surface, tens of microns are eliminated from the surface. During this process, sparks may be seen on the zirconia surface indicating high stress and temperature. Surface preparation with a coarse bur does not cause any chipping at the margins of zirconia ceramics but it may cause subsurface cracks and consequently decrease the strength ${ }^{23-25)}$.

In the current study, a high-speed handpiece with a diamond bur along with water and air spray was used in five back and forth motions, during which, the entire length of the bur was in contact with the ceramic surface. Roughening with diamond bur must be done at high speed to prevent hand piece vibration (since this may cause cracks at the ceramic margins) ${ }^{26,27)}$. The results showed that zirconia ceramic surface preparation with diamond bur, irrespective of the type of primer, yielded the highest bond strength. Derand et al. evaluated the bond strength of resin cements to zirconia ceramics and stated that grinding of the ceramic surface with diamond bur yielded a rougher surface than air abrasion and conse- quently resulted in higher bond strength ${ }^{28)}$. In our previous pilot study, bur preparation created a rough surface with parallel retentive grooves; whereas, sandblasting created non-uniform irregularities ${ }^{29}$. A previous study discussed that rougher surfaces had higher surface free energy and provided a larger bonding area ${ }^{30}$. Saygili et al. demonstrated that the shear bond strength of IPS Empress Blocks to resin cements increased after surface roughening by diamond bur and this value was higher compared to air abrasion and control groups ${ }^{24)}$. Bur preparation creates retentive grooves. Primers flow into these grooves and increase the bond strength of composite resin to ceramic ${ }^{31)}$.

We found that air abrasion, irrespective of the primer system used, increased the bond strength compared to the control group. Our results in this respect are in accord with those of Akyil et al, showing that air abrasion yielded higher bond strength than Er:YAG and Nd:YAG laser preparations and the control group ${ }^{10)}$. Also our results showed that the shear bond strength of air abrasion subgroups of both primers was significantly higher than that of laser subgroups ( $\mathrm{p}$ value $<0.05$ ). Although in our previous study ${ }^{29)}$, blocks irradiated with Nd:YAG laser with $1.5 \mathrm{~W}$ power showed high surface roughness in profilometry but in the present study this subgroup had the lowest shear bond strength. We concluded that different surface preparations differently affect the bond strength, and higher surface roughness does not necessarily increase the bond strength. A previous study reported the minimum clinically acceptable bond strength to be 10 $\mathrm{Mpa}^{1)}$. In the current study, monobond plus specimens that were subjected to Nd:YAG laser did not guarantee an optimal clinical service since they did not have high enough shear bond strength. Moreover, Nd:YAG laser, irrespective of the type of primer, yielded lower bond strength compared to the control group. The results of the current study are in line with those of Kasraei et al ${ }^{32)}$. They concluded that Nd:YAG laser irradiation decreased the bond strength compared to the control group. In another research, Nd:YAG irradiated zirconia blocks showed microcracks and large deep pits ${ }^{33)}$. Also several studies have been shown that inappropriate Nd:YAG laser output power decreases the bond strength between resin cement and zirconia substrate. They stated that overheating, the formation of microcracks and superficial damaged layer weaken the bond strength between resin cement and zirconia substrate ${ }^{7,33-35)}$.

Moreover, Er:YAG laser irradiated groups showed bond strengths similar to the control group. Cavalcanti et al. applied Er:YAG laser with $200 \mathrm{~mJ} /$ pulse energy and 10 $\mathrm{Hz}$ frequency for five seconds on the zirconia ceramic surface and reported that the bond strength did not increase compared to air abrasion, and it even decreased compared to the control group ${ }^{36)}$. Gomes et al. stated irregularities and erosions created by Er:YAG laser have in- 
sufficient micromechanical retention and result in limited penetration of the resin cement ${ }^{37}$. Other study explained that Er:YAG laser irradiation causes micro-explosions and creates debris that can strongly bond to ceramic surfaces and resin cements. This layer may weakly bond to the underlying surfaces and decrease the bond strength ${ }^{38}$.

Silveira et al. reported higher bond strength values after Nd:YAG laser irradiation of In-Ceram alumina compared to air abrasion and silica-coating ${ }^{39)}$. Spohr et al. irradiated In-Ceram Zirconia with Nd:YAG laser and obtained higher bond strength compared to air abrasion and the use of $\mathrm{SiO}_{2}$ particles ${ }^{40)}$. In the above-mentioned studies, zirconia surfaces had been covered with graphite powder to better absorb laser energy ${ }^{39}, 40$ ). In our study, graphite powder was not used. Differences in the results of these studies may be attributed to the application of graphite powder, different durations of laser irradiation and the use of different types of zirconia ceramics ${ }^{31}$.

According to our previous study we choose these laser parameters ${ }^{29)}$. There are some studies with other different wavelengths (1340 and $10600 \mathrm{~nm}$ ). El Gamat and et al. used $\mathrm{CO}_{2}$ laser at 10,600 nm wavelength. They concluded that $\mathrm{CO}_{2}$ irradiation increased shear bond strength between Emax ZirCAD and resin composite ${ }^{41)}$. Also, El Gamat showed that the micro hardness of Emax ZirCAD ceramic decreased after $\mathrm{Nd}$ :YAP and $\mathrm{CO}_{2}$ laser irradiation. They stated temperature had a significant effect on micro hardness ${ }^{42}$. Again in another research, El Gamat and et al. concluded that $\mathrm{Nd}$ :YAP and $\mathrm{CO}_{2}$ lasers could modify ceramic surfaces without chemical composition alteration 43). Also, Prieto M and et al. showed that laser treatment of zirconia with femtosecond laser improved the adhesion between zirconia and resin cement ${ }^{44)}$.

The minimum shear bond strength required by ISO standard is $5 \mathrm{MPa}$; however, the minimum clinically acceptable bond strength is $10-13 \mathrm{MPa}{ }^{45,46}$. Achieving a long-term bond requires adequate knowledge and thorough understanding of adhesive materials and surface preparation techniques. One of the most important factors required to obtain the highest bond strength is selection of a reliable bonding system.

Several studies have shown that air abrasion with aluminum oxide particles significantly affects the zirconia bond to resin cements containing functional phosphate monomers such as 10-MDP 15, 19, 47). In the current study, application of phosphate monomers on the surface of untreated zirconia caused a weak bond to composite. But, surface preparation with diamond bur and air abrasion significantly enhanced the bond strength. This indicates that mechanically created surface roughness is the main mechanism of bonding. Also, pairwise comparison of primers with different surface preparations showed that $\mathrm{Z}$ Prime Plus caused higher bond strength than monobond plus. However, this difference was only significant in the Nd:YAG laser subgroups. Z Prime Plus caused significantly higher bond strength than monobond plus ( $\mathrm{p}$ value $\leq$ 0.05). Z Prime Plus is a combination of organophosphate monomers and carboxylic acid. Organophosphate monomers, similar to silane, have two parts of organofunctional and phosphate monomers. The organofunctional part is often a methacrylate group that can copolymerize with monomers of the composite resin. Phosphate monomers also contain phosphoric acid groups that can bond to metal oxides in Y-TZP substrate. Carboxylic acid monomers also enhance the bond strength ${ }^{48}$. Monobond plus contains 10-MDP phosphate monomer, sulphide methacrylate and silane methacrylate (table 1). Several studies have shown that application of primers containing phosphate monomers such as 10-MDP or 6-MHPA increases the bond strength of resin cement to oxide ceramics ${ }^{14,49,50)}$

A passive layer of zirconium oxide covers the zirconia ceramic surface. Thus, chemical properties of this ceramic surface are similar to those of metal surfaces. Hydroxyl groups may be present on the zirconia ceramic surface and resin cements with any polymer or monomer with polar functional groups may be capable of chemically bonding to polar hydroxyl groups on the zirconia ceramic surface ${ }^{51)}$. Thus, both primer systems used in this study are expected to have the potential to chemically bond to zirconia ceramic due to having polymers and functional monomers. Also, 10-MDP is structurally a phosphate ester monomer containing a $\mathrm{P}=\mathrm{O}$ group and two hydroxyl groups in a molecule. Since these primers have slight amounts of water, most MDPs do not break down in spite of their acidic nature. Hydroxyl and $\mathrm{P}=\mathrm{O}$ groups participate in hydrogen bonds on the zirconia surface. The non-polar spacer group in 10-MDP comprises of a long saturated carbon chain and distances the water molecules from the hydrogen bond formed at the zirconia-composite interface. Carbon-carbon double bonds at the end of 10-MDP molecules copolymerize with resin monomers ${ }^{51,52)}$. Our results approved our hypothesis to some extent since the type of preparation significantly affected the composite bond to zirconia. The lowest bond strength was noted in monobond plus Nd:YAG laser subgroup and the highest in the $\mathrm{Z}$ Prime Plus bur preparation subgroup. But, the type of primer in most subgroups had no significant effect on bond strength. Clinically, bur preparation method does not require purchase of equipment as do air abrasion and laser. Moreover, bur preparation is cost effective and efficient. Thus, based on the results and considering the greater accessibility and availability of bur compared to air abrasion, diamond bur along with water coolant is recommended for surface preparation of zirconia ceramics.

\section{Conclusions}

- Type of surface preparation significantly affected the bond strength. 
- Surface preparation with Nd:YAG laser showed the lowest bond strength.

- Er:YAG laser irradiated samples showed lower bond strength than air abraded and control samples.

- Type of primer had no significant effect on bond strength and the bond strength was equal in all subgroups of the two primers except for Nd:YAG laser sub-

\section{References}

1: Kim BK, Kyung Bae HE, Shim J, Woo L (2005). The influence of ceramic surface treatments on the tensile bond strength of composite resin to all-ceramic coping materials. J Prosthet Dent, 94:357-62

2: Sailer I, Fehér A, Filser F, Gauckler LJ, Lüthy H, Hämmerle CH (2007). Five-year clinical results of zirconia frameworks for posterior fixed partial dentures. Int J Prosthodont, 20:383-8.

3: Özcan M, Valandro LF, Amaral R, Leite F, Bottino MA (2009). Bond strength durability of a resin composite on a reinforced ceramic using various repair systems. Dental materials, 25: $1477-1483$

4: Güler AU, Yilmaz F, Ural C, Güler E (2005). Evaluation of 24-hours shear bond strength of resin composite to porcelain according to surface treatment. Int J Prosthodont, 18 (2): $156-60$

5: Filho AM, Vieira LC, Araújo E, Monteiro Júnior S (2004). Effect of different ceramic surface treatments on resin microtensile bond strength. J Prosthodont, 13(1):28-35.

6: Della Bona A, Borba M, Benetti P, Cecchetti D (2007). Effect of surface treatments on the bond strength of a zirconia reinforced ceramic to composite resin. Braz Oral Res, 21(1):10-15

7: KoizukaM, Komine F, BlatzMB, Fushiki R, Taguchi K, Matsumura H (2013). The effect of different surface treatments on the bond strength of a gingiva-colored indirect composite veneering material to three implant framework materials. Clin Oral Impl Res, 24: 977-984

8: Chen L, Suh BI, Kim J, Tay FR (2011). Evaluation of silica-coating techniques for zirconia bonding. Am J Dent, 24(2):79-84.

9: Futoshi Komine, Ryosuke Fushiki, Mai Koizuka, Kohei Taguchi,Shingo Kamio, Hideo Matsumura (2012). Effect of surface treatment on bond strength between indirect composite material and a zirconia framework. Journal of Oral Science, 54(1): 39-46.

10: Akyıl M, Uzun H, Bayındır F (2010). Bond Strength of Resin Cement to Yttrium-Stabilized Tetragonal Zirconia Ceramic Treated with Air Abrasion,Silica Coating and Laser Irradiation . Photomedicine and Laser Surgery, 28(6): 801-808

11: Minamizato T (1990). Slip-cast zirconia dental roots with tunnels drilled by laser process. J Prosthet Dent, 63(6):67784.

12: Shiu P, Souza-Zaroni WC, Eduardo CdeP, Youssef MN (2007). Effect of feldspathic ceramic surface treatments on bond strength to resin cement. Photomed Laser Surg, 25(4):291-6

13: Ersu B, Yuzugullu B, Yazici AR, Canay S. Surface roughness and bond strengths of glass-infiltrated alumina-ceramics prepared using various surface treatments. journal of dentistry 2009;37: $848-856$

14: Tsuo Y, Yoshida K, Atsuta M (2006). Effects of Alumina-blasting and Adhesive Primers on Bonding between Res- groups.

- Z Prime Plus Nd:YAG laser subgroup showed higher bond strength than monobond plus Nd:YAG laser subgroup.

- Surface roughening by diamond bur yielded the highest bond strength among all subgroups.

in Luting Agent and Zirconia Ceramics. Dental Materials Journal, 25(4):669-674.

15: Kitayama Sh, Nikaido T, Takahashi R, Zhu L, Ikeda M, Richard M. Foxton R, Sadr A, Tagami J (2010). Effect of primer treatment on bonding of resin cements to zirconia ceramic. Dental materials, 26:426-432

16: Fischer J, GroHmann Ph, Stawarczyk B (2008). Effect of zirconia surface treatment on the shear strength of zirconia/veneering ceramic composites. Dental materials journal, 27(3): 448-54

17: Queiroz J.R, Paulo GP, Ozcan M, Nogueiro L (2012). Effect of airborne particle abrasion protocols on surface topography of Y-TZP ceramic. Ceramica, 58:253-61

18: Khoroushi M, Motamedi Sh (2007). Shear Bond Strength of Composite-Resin to Porcelain: Effect of Thermocycling. Journal of Dentistry, Tehran University of Medical Sciences, 4(1): 21-26

19: Özcan M, Nijhuis H, Valandro Lf (2008). Effect of Various Surface Conditioning Methods on the Adhesion of Dual-cure Resin Cement with MDP Functional Monomer to Zirconia after Thermal Aging. Dental Materials, 27(1):99-104

20: Aboushelib MN, Mirmohamadi H, Matinlinna JP, Kukk E, Ounsi HF, Salameh Z (2009). Innovations in bonding to zirconia-based materials. Part II: Focusing on chemical interactions. Dental Materials, 25(8):989-93.

21: B.Blatz M, Sadan A, Kern M (2003). Resin-ceramic bonding: a review of the literature. J Prosthet Dent, 89:268-74

22: Gandolfi Paranhos MP, Burnett L H, Magne P (2011). Effect of $\mathrm{Nd}$ :YAG laser and $\mathrm{CO}_{2}$ laser treatment on the resin bond strength to zirconia ceramic. Quintessence Int, 42:79-89

23: Yongqi Zh, Li J, Jingchao Zh, Yunmao L, Junjun L, Wei L (2012). Influence of different surface treatments on computer-aided design and manufacturing (CAD-CAM) zirconia frameworks. Scientific Research and Essays, 7(8):957-962

24: Saygili G, Ahmali S (2003). Effect of ceramic surface treatment on the shear bond strengths of two resin luting agents to all-ceramic materials. Journal of Oral Rehabilitation, 30: 758-764

25: Kern M, Barloi A, Yang B (2009). Surface Conditioning Influences Zirconia Ceramic Bonding. J Dent Res, 88(9):817-822

26: Reston EG, Filho SC, Arossi G, Cogo RB, Rocha CS, Closs LQ (2008). Repairing Ceramic Restorations: Final Solution or Alternative Procedure? Operative Dentistry, 33(4): 461-466

27: Kussano C, Bonfante G, Batista J, Nogueira Pinto J (2003). Evaluation of Shear Bond Strength of Composite to Porcelain According to Surface Treatment. Braz Dent J, 14(2): 132 135

28: Derand P, Derand T (2000). Bond strength of luting cements to zirconium oxide ceramics. Int J Prosthodont, 13:131-5

29: Arami S,Hasani T M, Namdar S F, Chiniforoush N (2014). Effects of different lasers and particle abrasion on surface characteristics of zirconia ceramics. journal of dentistry , Tehran university of medical sciences, 11(2): 233-241 
30: Yamaguchi H, Satoshi Ino S, Hamano N, Okada Sh, Teranaka T (2012). Examination of bond strength and mechanical properties of Y-TZP zirconia ceramics with different surface modifications. Dental Materials Journal, 31(3): 472-480

31: Subaşı MG, İnan Ö (2012). Evaluation of the topographical surface changes and roughness of zirconia after different surface treatments. Lasers in medical science, 27(4):735-42.

32: Kasraei S, Rezaei-Soufi L, Yarmohamadi E, Shabani A (2015). Effect of $\mathrm{CO}_{2}$ and Nd:YAG Lasers on Shear Bond Strength of Resin Cement to Zirconia Ceramic. Journal of Dentistry (Tehran, Iran), 12(9):686-694.

33: Keshvad, A. and Hakimaneh, S. M. R. (2018), Microtensile Bond Strength of a Resin Cement to Silica-Based and Y-TZP Ceramics Using Different Surface Treatments. Journal of Prosthodontics, 27: 67-74.

34: Akın H, Ozkurt Z, Kirmalı O, Kazazoglu E, Ozdemir AK (2011). Shear bond strength of resin cement to zirconia ceramic after aluminum oxide sandblasting and various laser treatments.Photomed Laser Surg, 29(12):797-802.

35: Lin Y, Song X, Chen Y, Zhu Q, Zhang W (2013). Effect of Er:YAG laser irradiation on bonding property of zirconia ceramics to resin cement. Photomed Laser Surg, 31(12):619-25.

36: Cavalcanti AN, Foxton RM, Watson TF, Oliveira MT, Giannini M, Marchi GM (2009). Bond Strength of Resin Cements to a Zirconia Ceramic with Different Surface Treatments. Operative Dentistry, 34(3): 280-287

37: Ana Luísa Gomes, João Carlos Ramos, Sergio Santosdel Riego, Javier Montero, Alberto Albaladejo (2015). Thermocycling effect on microshear bond strength to zirconia ceramic using Er:YAG and tribochemical silica coating as surface conditioning. Lasers in Medical Science,30(2): 787-795

38: Subaşı MG, İnan Ö (2014). Influence of surface treatments and resin cement selection on bonding to zirconia. Lasers in medical science, 29(1):19-27.

39: Silveira Bd, Paglia A, Burnett H, Shinkai R, Eduardo C, Spohr A (2005). Microtensile bond strength between resin cement and an aluminous ceramic treated with Nd:YAG laser, Rocatec system or aluminum oxide sandblasting. Photomed Laser Surgery, 1(23): 543-548

40: Spohr A, Borges G, Burnett 1, Goncalves E, Silva H (2008). Surface Modification of In-Ceram Zirconia Ceramic by Nd:YAG Laser, Rocatec System or Aluminum oxide sandblasting and its Bond Strength to a Resin Cement. Photo- medicne and Laser Surgery, 26(1):203-208

41: El Gamal A, Medioni E, Rocca JP, Fornaini C, Muhammad OH, Brulat-Bouchard N (2017). Shear bond, wettability and AFM evaluations on $\mathrm{CO}_{2}$ laser-irradiated CAD/CAM ceramic surfaces. Lasers in medical science, 32(4):779-85.

42: El Gamal A, Rocca JP, Fornaini C, Medioni E, Brulat-Bouchard N (2017). Microhardness evaluations of CAD/CAM ceramics irradiated with $\mathrm{CO}_{2}$ or Nd: YAP laser. Laser therapy, 26(1):13-8.

43: El Gamal A, Fornaini C, Rocca JP, Muhammad OH, Medioni E, Cucinotta A, Brulat-Bouchard N (2016). The effect of $\mathrm{CO}_{2}$ and Nd: YAP lasers on CAD/CAM Ceramics: SEM, EDS and thermal studies. Laser therapy, 25(1):27-34.

44: Prieto MV, Gomes AL, Martín JM, Lorenzo AA, Mato VS, Martínez AA (2016). The Effect of Femtosecond Laser Treatment on the Effectiveness of Resin-Zirconia Adhesive: An In Vitro Study. Journal of lasers in medical sciences, 7(4):214.

45: International Standardization Organization, ISO 10477 Dentistry -Polymer-Based Crown and Bridge Materials, Amendment (1996)

46: Thurmond J, Barkmeier W, Wildwerding M (1994). Effect of porcelain surface treatments on bond strengths of composite resin bonded to porcelain. J Prosthet Dent, 72:355-359

47: Yang B, Barloi A, Kern M (2010). Influence of air-abrasion on zirconia ceramic bonding using an adhesive composite resin. dental materials, $26: 44-50$

48: Pott P-C., Stiesch M, Eisenburger M (2015).Influence of 10MDP Adhesive System on Shear Bond Strength of Zirconia-Composite Interfaces. J Dent Mater Tech, 4(3): 117-26

49: Chen Ch, Xie H, Song X, Burrow M F, Chen G, Zhang F (2014). Evaluation of a Commercial Primer for Bonding of Zirconia to Two Different Resin Composite Cements. J Adhes Dent, 16: 169-176

50: Chen L, Suh BI, Brown D, Chen X (2012).Bonding investigation of primed zirconia ceramics for evidence of chemical bonding and improved bond strength. American Journal of Dentistry, 25:103-8

51: Amarel M, Belli R,Cesar P F,Valandro L F, Petschelt A, Lohbauer U (2014). The potential of novel primers and universal adhesives to bond to zirconia. J of dentistry, 42:90-98

52: Kim MJ, KimYK, Kim KH, Kwon TY (2011). Shear bond strengths of various luting cements to zirconia ceramic: Surface chemical aspects. Journal of dentistry, 39: 795-803

\section{Acknowledgment}

The results presented in this study have been taken in part from a postgraduate thesis in Laser Research Center of Dentistry, Dentistry Research Institute, Tehran University of Medical Sciences, Iran.

No author has any conflicts of interest (COI). 